\title{
FOLIATIONS AND THE TOPOLOGY OF 3-MANIFOLDS ${ }^{1}$
}

\author{
BY DAVID GABAI
}

In this announcement we discuss the close relationship between the topology of 3-manifolds and the foliations that is possesses. We will introduce and state the main result, then use it and the ideas of its proof to state some geometric and topological corollaries. Details to almost all the results can be found in $\left[\mathbf{G}_{\mathbf{4}}\right]$.

Given a compact, connected, oriented 3-manifold, when does there exist a codimension-1 transversely oriented foliation $₹$ which is transverse to $\partial M$ and has no Reeb components? If such an $\mathcal{F}$ exists $\partial M$ necessarily is a (possibly empty) union of tori and $M$ is either $S^{2} \times S^{1}$ (and $\mp$ is the product foliation) or irreducible. The first condition follows by Euler characteristic reasons while the latter basically follows from the work of Reeb [Re] and Novikov $[\mathbf{N}]$ although first observed by Rosenberg [Ro]. Our main result says that such conditions are sufficient when $H_{2}(M, \partial M) \neq 0$.

If such a foliation $\mathcal{F}$ exists on $M$ then it follows from the work of Thurston $\left[\mathbf{T}_{\mathbf{1}}\right.$ ] that any compact leaf $L$ is a Thurston norm minimizing surface [i.e., $\left|\chi\left(L^{\prime}\right)\right| \leq\left|\chi\left(T^{\prime}\right)\right|$ for any properly embedded $T$ with $[T]=[L] \in H_{2}(M, \partial M)$ (or $H_{2}(M)$ if we were discussing the norm on $H_{2}(M)$ ), where $S^{\prime}$ denotes $S$-(sphere and disc components)] for the class $[L] \in H_{2}(M, \partial M)$. Our main result says that for a 3-manifold $M$ satisfying the above necessary conditions any norm minimizing surface can be realized as a compact leaf of a foliation without Reeb components.

THEOREM. Let $M$ be a compact, connected, irreducible, oriented 3-manifold whose boundary is a (possibly empty) union of tori i.e., $M$ is Haken and $\chi(M)=$ 0 . Let $S$ be any norm minimizing surface representing a nontrivial class $z \in$ $H_{2}(M, \partial M)$. Then there exists foliations $\mathcal{F}_{0}$ and $\mathcal{F}_{1}$ of $M$ such that

(1) for $i=0,1, \mathcal{F}_{i} \pitchfork \partial M$ and $\mathcal{F}_{i} \mid \partial M$ has no Reeb components,

(2) every leaf of $\mathcal{F}_{0}$ and $\mathcal{F}_{1}$ nontrivially intersects a closed transverse curve,

(3) $S$ is a compact leaf of both $\mathcal{F}_{0}$ and $\mathcal{F}_{1}$,

(4) $\mathcal{F}_{0}$ is of finite depth,

(5) $\mathcal{F}_{1}$ is $C^{\infty}$ except possibly along toral components of $S$.

COROLLARY. Let $L$ be an oriented nonsplit (i.e., no embedded $S^{2}$ in $S^{3}-L$ separates the components of $L$ ) link in $S^{3}$. Then $S$ is a surface of minimal genus for $L$ if and only if there exists a $C^{\infty}$ transversely oriented foliation $\mathcal{F}$ of $S^{3}-N(L)$ such that

Received by the editors August 16, 1982.

1980 Mathematics Subject Classification. Primary 57R30, 57N10.

${ }^{1}$ Partially supported by NSF Grant MCS-80-17200. (C) 1983 American Mathematical Society
$0273-0979 / 82 / 0000-1038 / \$ 01.75$ 
(1) $₹ \pitchfork \partial N(L)$ and $\mathcal{F}$ and $\mathcal{F} \mid \partial N(L)$ have no Reeb components,

(2) $S$ is a compact leaf.

The $\Rightarrow$ direction follows from the theorem and Thurston $\left[\mathbf{T}_{\mathbf{1}}\right]$ proved the converse. Simple methods for explicitly constructing foliations for certain classes of knots and links, thereby computing their genera, can be found in $\left[\mathbf{G}_{1}\right.$ and $\mathbf{G}_{2}$ ].

COROLlarY. A nontrivial link $L$ in $S^{3}$ is nonsplit if and only if $L$ is the set of cores of Reeb components of some foliation $₹$ of $S^{3}$.

The $\Rightarrow$ direction follows from the theorem and Novikov [N] proved the converse in 1963 thereby answering the question of Laudenbach and Roussarie [L-R] which asked which links could be realized as cores of Reeb components of foliations of $S^{3}$.

By understanding how to 'cut and paste' foliations we have the following

COROLlARY. If for $i=1,2, R_{i}$ is a surface of minimal genus for the oriented link $L_{i} \subset S^{3}$ and $R$ is any Murasugi Sum $[\mathbf{M}]$ or $\left[\mathbf{G}_{3}\right]$ (or generalized plumbing) of $R_{1}$ and $R_{2}$ with $L=\partial R$, then $R$ is a minimal genus surface for the oriented link $L$. Conversely, if $R$ is a surface of minimal genus for $L$, then for $i=1,2, R_{i}$ is a surface of minimal genus for $L_{i}$.

This generalizes the classical result due to Seifert in the 1930s that the connected sum of minimal genus surfaces is a surface of minimal genus and conversely.

By understanding the construction explicitly and knowing how to perturb foliations we have the

COROLlARY. Let $M \neq D^{2} \times S^{1}$ be a Haken 3-manifold with $\chi(M)=0$. If $\mathrm{H}_{2}(M, \partial M)$ is not generated by tori and annuli, then there exists a $C^{\infty}$ transversely oriented foliation $\mathcal{F}$ on $M$ such that $\mathcal{F} \pitchfork \partial M, \mathcal{F} \mid \partial M$ has no Reeb components and no leaf of $\mathcal{F}$ is compact.

In particular we have the

COROLLARY. Let $M$ be either a compact, connected, oriented 3-manifold whose interior has a complete hyperbolic metric and $H_{2}(M, \partial M) \neq 0$ or $M=$ $S^{3}-\stackrel{\circ}{N}(L)$ where $L$ is a nonsplit nontrivial link in $S^{3}$. Then there exists a $C^{\infty}$ transversely oriented foliation $\mathcal{F}$ of $M$ such that $\mathcal{F}$ has no compact leaves, $\mp \pitchfork \partial M$ and $\mathcal{F} \mid \partial M$ has no Reeb components.

Applying a criterion of Sullivan [Sn] we obtain the

Corollary. Suppose $M$ is a Haken 3-manifold with $\chi(M)=0$ and $H_{2}(M, \partial M) \neq 0$. Then there exists a Riemannian metric and foliation $\mathcal{F}$ on $M$ such that $\mathcal{F} \pitchfork \partial M$ and every leaf is minimal (i.e. has mean curvature 0 ).

Since foliations 'pull back' nicely we have the

COROLLARY. Let $M$ be a compact oriented 3-manifold. Let $p: \tilde{M} \rightarrow M$ be an $n$-fold covering map and let $z \in H_{2}(M)=H^{1}(M, \partial M)$ or $z \in H_{2}(M, \partial M)=$ $H^{1}(M)$. Then $n(x(z))=x\left(p^{*}(z)\right)$ where $x(z)$ denotes the Thurston norm of $z$. 
This was conjectured by Thurston in $\left[\mathbf{T}_{\mathbf{1}}\right]$ as was the next

COROLlARY. Let $M$ be a compact oriented 3-manifold; then $x_{s}=x$ where $x_{s}$ denotes the norm on homology based on singular surfaces. Equivalently the Gromov norm $\left[\mathbf{T}_{\mathbf{2}}, \S 6\right]$ on $\mathrm{H}_{2}(M)$ or $\mathrm{H}_{2}(M, \partial M)$ is equal to twice the Thurston norm.

To prove this we first reduce to the case when $M$ is closed and irreducible. By the theorem a homology class can be represented as a compact leaf of a finite depth foliation $\mathcal{F}$ without Reeb components. We then generalize Thurston's proof that such leaves are norm minimizing in the Thurston norm to the singular norm.

The last Corollary is a version of Dehn's lemma for higher genus surfaces. In words it says that given an immersed surface in $M$ there exists an embedded surface of no larger "topological complexity" which represents the same homology class. This answers a question of Papakyriakopoulos $[\mathbf{P}]$.

Recall that the immersed genus of a knot $K$ in $S^{3}$ is the smallest $g$ such that $K$ bounds a punctured immersed surface $S$ of genus $g$ which is nonsingular along the boundary, i.e. $f: S \rightarrow S^{3}$ and $f^{-1}(K)=\partial S$. A special case of the last Corollary is the

COROLLARY. If $K$ is a knot in $S^{3}$, then the immersed genus of $K$ equals the genus of $K$.

Understanding finite depth foliations yields the following result.

Define an equivalence relation on 3-manifolds generated by the operation of splitting and regluing along incompressible surfaces.

COROLLARY. If $M$ is Haken and $\chi(M)=0$, then $M$ is equivalent to exactly one of

(a) torus $\times S^{1}$,

(b) 2-hole torus $\times S^{1}$,

(c) $\operatorname{disc} \times S^{1}$,

(d) annulus $\times S^{1}$,

(e) punctured torus $\times S^{1}$.

\section{BIBLIOGRAPHY}

[G $\left.\mathbf{G}_{1}\right]$ D. Gabai, Foliations and genera of links, preprint.

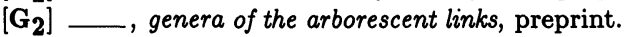

[G] _- The Murasugi sum is a natural geometric operation, preprint.

$\left[\mathbf{G}_{\mathbf{4}}\right] \longrightarrow$, Foliations and the topology of 3-manifolds, preprint.

[L-R] F. Laudenbach and R. Roussarie, Un exemple de feuilletage sur $S^{3}$, Topology 9 (1970), 63-70.

[M] K. Murasugi, On a certain subgroup of an alternating link, Amer. J. Math. 85 (1963), 544-550.

[N] S. P. Novikov, Topology of foliations, Trans. Moscow Math. Soc. 14 (1965), 268-305.

[P] C. D. Papakyriakopoulos, On Dehn's Lemma and the asphericity of knots, Ann. of Math. (2) 66 (1957), 1-26.

[Re] S. Reeb, Sur certaines propriétés topologiques des variétés feuilletées, Actualités Sci. Indust. No. $1183=$ Publ. Inst. Math. Univ. Strasbourg, no. 11, Hermann, Paris, 1952, pp. 91-158.

[Ro] H. Rosenberg, Foliations by planes, Topology 7 (1968), 131-138. 
[Sn] D. Sullivan, A homological characterization of foliations consisting of minimal surfaces, Comment. Math. Helv. 54 (1979), 218-223.

[ $\left.\mathbf{T}_{\mathbf{1}}\right] \mathrm{W}$. Thurston, $A$ norm on the homology of 3-manifolds, preprint.

$\left[\mathbf{T}_{2}\right]$ _, The topology and geometry of 3-manifolds, Princeton notes.

Department of MAThematics, HaRVARd University, CAMBridge, MassACHUSETTS 02138

Current address: School of Mathematics, Institute for Advanced Study, Princeton, New Jersey 08540 